\title{
An Approach to the Conversion of the Power Generated by an Offshore Wind Power Farm Connected into Seawave Power Generator
}

\author{
Vincenzo Franzitta $^{1}$, Antonio Messineo $^{2, *}$ and Marco Trapanese ${ }^{3}$ \\ ${ }^{I}$ Department of Energy and Environmental Researches, University of Palermo, Italy \\ ${ }^{2}$ Faculty of Engineering \& Architecture, Kore University of Enna, Italy \\ ${ }^{3}$ Electrical Engineering Department, University of Palermo, Italy
}

\begin{abstract}
In this paper we present an approach to the conversion of the power generated by a seawave power generator integrated in an offshore wind power farm. The article is focused on low power generators that could be used in offshore installations. The generator is a Permanent Magnet (PM) linear generator that generates highly distorted emfs. We propose and compare two possible ways to connect the generator to the network and to the offshore wind power farm. One is based on an approach where for each generator there is a conversion subsystem that permits the direct connection of the generator to the a.c. network, the other one is based on an a.c.-d.c. converter that is connected to the generator, the converter outputs on a dc link that can receive the power from other units and that can supply a d.c.-a.c. converter directly connected to the main.
\end{abstract}

Keyword: Seawave, power generator, offshore wind farm, emfs, a.c. network.

\section{INTRODUCTION}

Amongst renewable energy sources, sea waves are definitely the one that has been less utilized. This is probably caused by three reasons: i) the low energy concentration in the sea waves, at least in the sites where it is technically possible to install conversion facilities; ii) the strong technical novelties that are required to extract the energy that is contained in a highly and stochastically variable motion; iii) the difficulty to find a suitable electrical system able to convert the energy contained in the motion of the sea into electrical energy.

Up to now, the main approach, that has been followed to exploit the energy of sea waves, uses Well's turbines in oscillating water columns (OWC). Recently, the use of linear generator has been proposed to extract energy from sea waves [1-9].

In this approach the slider of a linear generator is directly coupled to a device that is vertically moved from the sea waves [1-10]. According to the conversion strategy chosen, this device can be a buoyant or an air filled chamber completely submerged. Regardless to which strategy is chosen, wave energy conversion produces high force and low reciprocating speed.

Moreover, the behavior of both of them is highly stochastic because it is caused by the sea waves, which are essentially stochastic. As a result, the produced emf is highly distorted and can have a frequency very different from the frequency of the grid. Moreover, the natural way to design a

*Address correspondence to this author at the Faculty of Engineering \& Architecture, Kore University of Enna, Italy; Tel: +39 091238 61908; Fax: +39091 484425; E-mail: antonio.messineo@unikore.it seawave power farm is to integrate it to an offshore wind farm.

As a result, as far as a power plant composed from several seawave generator and several wind turbines is concerned, the connection of each of this generator to the grid is a formidable task that must be solved in order to make industrially feasible the production of electric power from sea waves. In this paper we propose two possible approaches and we compare each of them $[13,14]$.

In this paper, we present a study on the interconnection of a small set of wind turbines together with a seawave power generator.

This paper is organized as follows: in section 2 we present the sea wave generator and the operating principle of a sewave generator, in section 3 we show the emf produced from a seawave generator, in section 4 we present the two possible connection solutions and in section 5 we draw the conclusions.

\section{THE SEA WAVE GENERATOR}

It is assumed that the conversion system has the structure of Fig. (1) $[9,11,12]$. Other conversion topologies can be realized but the one of Fig. (1) captures the essential features of the most of conversion systems.

In this device the slider of the PM linear generator is driven from a buoyant (that is rigidly connected to the slider) and counteracted by a spring.

The motion of the slider can be modelled as follows:

$e(t)=i R_{i}+L \frac{\partial i}{\partial t}+i R$ 


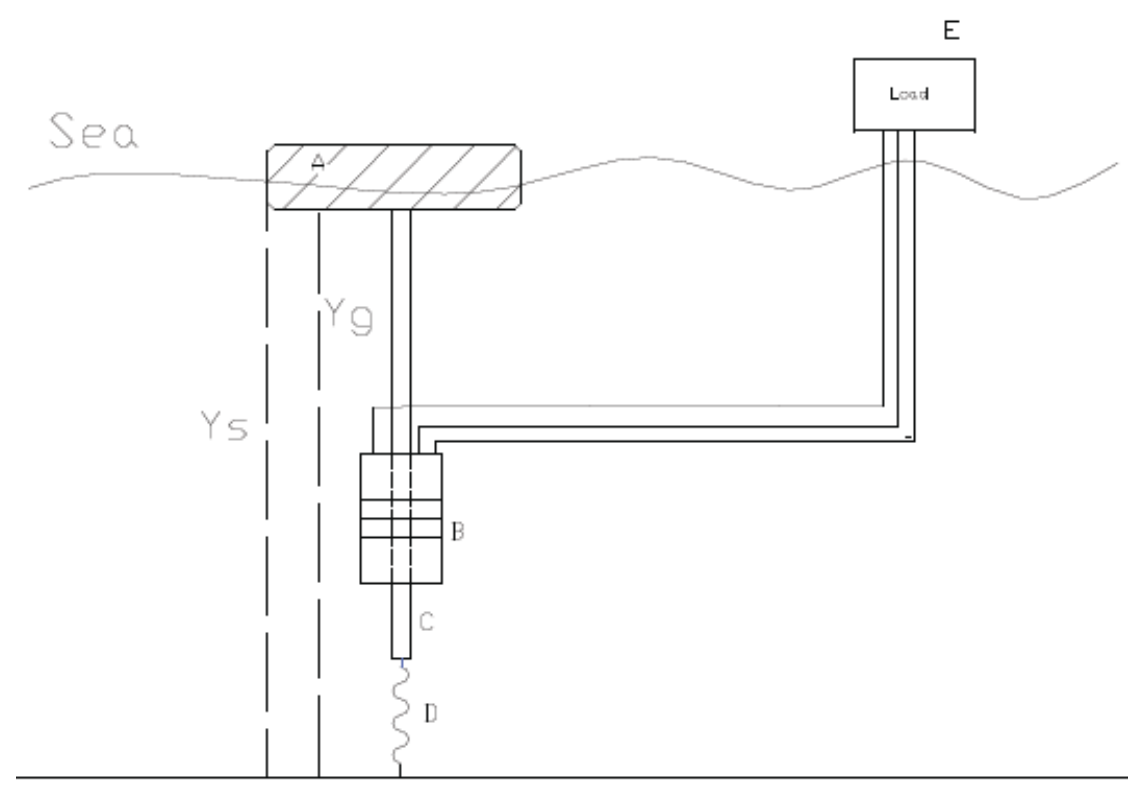

Fig. (1). Schematic description of a conversion system based on a PM linear generator. A is the buoyant, B is the PM linear generator, C is the slider, D is the spring and $\mathrm{E}$ is the electrical load.

$\mathrm{e}(\mathrm{t})=-\frac{\mathrm{d} \varphi}{\mathrm{dt}}$

$\rho g S\left(y_{s}-y_{g}\right)-m g-k i B-k_{s}\left(y_{g}-y_{0}\right)=m \frac{d^{2} y_{g}}{{d t^{2}}^{2}}$

Where $g$ is the acceleration gravity, $\rho$ is the water density, $S$ is the area of the buoyant, $y_{s}$ is the vertical coordinate describing the sea level, $y_{g}$ is the vertical coordinate describing the position of the slider, $k_{s}$ is the spring constant, $m$ is total mass of the translator and of the buoyant, $k$ is the electromagnetic constant, $i$ the current, $B$ the magnetic induction, $e(t)$ is the induced electromotive force (emf), $R_{i}$ is the internal resistance of the machine, $L$ is the inductance of the machine, $R$ is the resistance of the external load, $\varphi$ is the linked flux and $y_{0}$ is the rest position. The definition of the coordinates can be seen in Fig. (1).

The first equation models the dynamic behaviour of the device. On the left hand side there are the forces acting on the slider and on the right hand side there is the acceleration of the slider. No hydraulic friction is considered.

The second equation describes in terms of lumped parameters the generator connected to a resistive load. The third equations describes the emf.

Several assumptions have been done:

- the mathematical model used is one-dimensional;

- no losses are assumed in the hydraulic conversion systems;

- the losses in the electromagnetic generator are described by a single resistance;

- the external load is considered purely resistive (in fact the external load will be buffered by a power electronics device).

\section{THE EMF PRODUCED FROM THE SEA WAVE GENERATOR}

As it can be seen from (1)-(3) the emf is produced from the motion of the vertical level of the sea. The typical frequency of sea waves is in the range of few parts of $\mathrm{Hz}$ as a result a machine with a high number of poles is needed in order to obtain an output whose average frequency is in a range that is easily convertible.

However, even with this approach the emf will be highly not sinusoidal, stochastic with a high degree of impulsivity. In Fig. (2) an experimental emf produced such a linear generator is shown.

\section{THE POSSIBLE CONNECTION SOLUTION}

The conversion of a waveform such as the one in Fig. (2) into an a.c. waveform injectable into the grid is not an easy task. This task is also made more difficult from the fact that in a seawave power farm many generator will be present and each one will generate a voltage very distorted, synchronized nor to the other ones neither to the grid and, in addiction the wind farm tends to perturbate significantly the grid making even more difficult the interconnection procedures.

Moreover, the average frequency of each output emf will be different from the other ones. As a result an approach to the conversion of the output and to the connection of the generators to the grid must be found. We have examined two approaches:

1. a decentralized approach;

2. a partially centralized approach.

The decentralized approach converts from the distorted input to an a.c. output directly from the output of each machine. The typical structure of the converter is an ac-ac converter shown in Fig. (3a).

As a result each machine is directly connected to the grid through this a.c. converter. If the d.c. link voltage falls under 


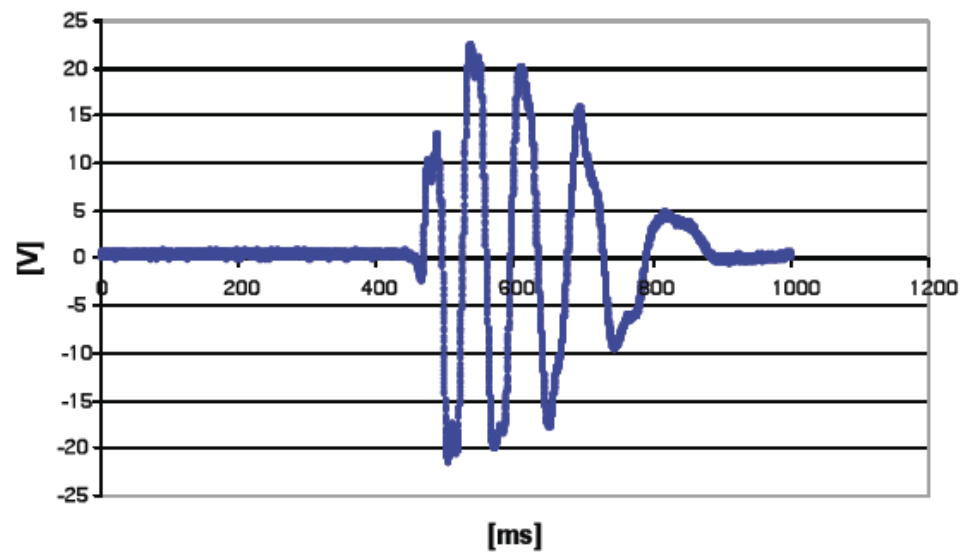

Fig. (2). No load emf in a single phase PM linear generator. The slider speed is $1.6 \mathrm{~m} / \mathrm{s}$.

A)
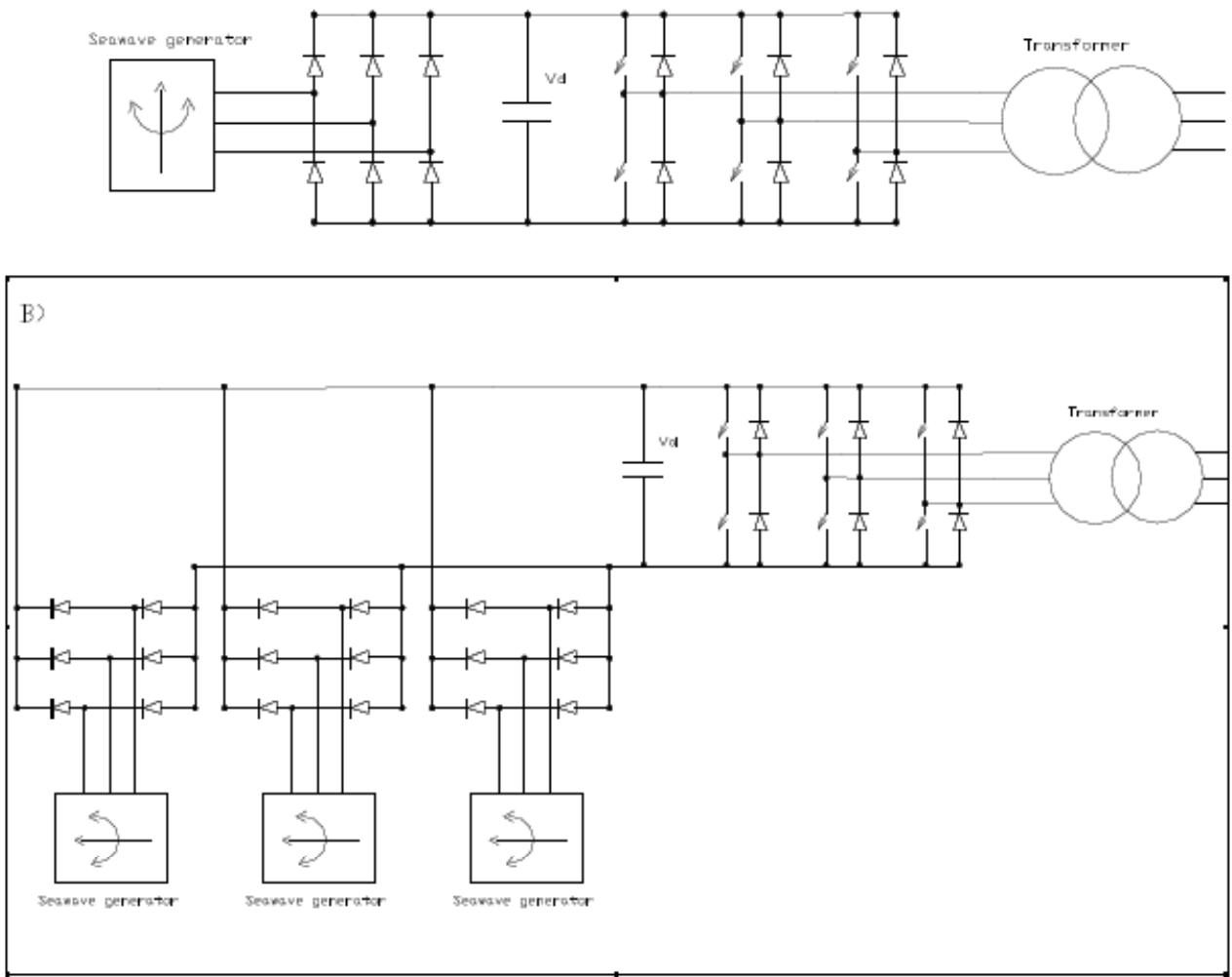

Fig. (3). a) Decentralized approach to the interconnection of a seawave generator to the grid; b) Partially centralized approach to the interconnection of several seawave generators (3) to the grid. In both cases between the transformer and the grid there is a filter that is not depicted in this Figure.

a critical values the generator must be disconnected from the grid because it does not input any power. As a result the buffer capacitance plays a role and should be used in an optimal way.

The partially centralized approach split the distorted voltage - d.c. conversion from the d.c.-a.c. conversion (Fig. 3b). It leaves the rectifiers on each single generator and connect all the outputs of the rectifiers to a common d.c. bus.

If the d.c. link voltage falls under a critical values the generator must be disconnected from the grid because it does not input any power. As a result the buffer capacitance plays a role and should be used in an optimal way. On the con- trary, the inverter is centralized and use as d.c. link the bus where all the generators are connected.

In order to compare the two approaches we have examined the behaviour of some parameters in a numerical simulation performed by simulink of a seawave powerfarm composed by 10 generators integrated to a wind farm composed by 5 turbines. The converter were simulated by assuming a standard numerical model of the inverter. The parameters that we have considered are the ripple in dc link, the time of use of each inverter the continuity of the generation. The simulation was run 5 times and in each time each generator was exposed to a set of waveform generated as illustrated in [2]. Each generator had the characteristics of the one re- 


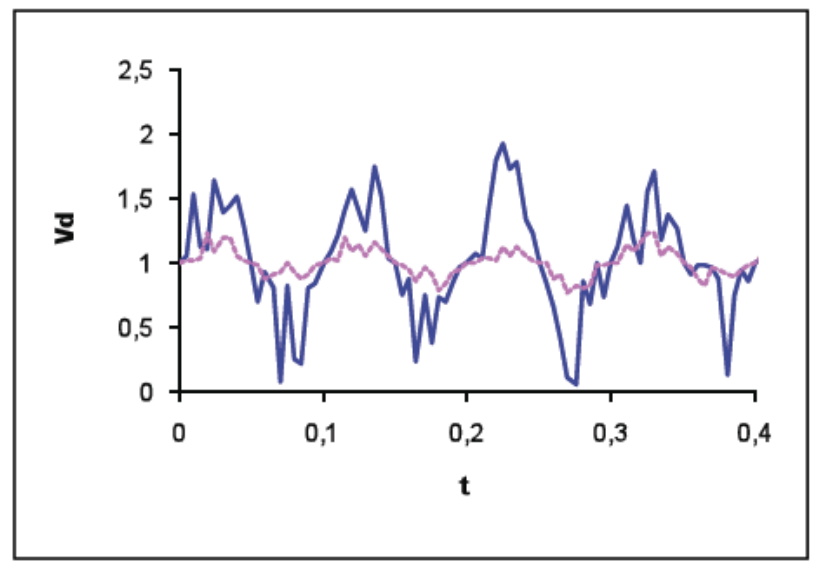

Fig. (4). Voltage ripple of the dc link (continuos line decentralized approach dotted line semi centralized approach).

ported in [2]. The rated power of the wind generators is in the order of kwatts.

In Fig. (4) the ripples of the dc link for the two approaches are compared. It can be clearly seen that in all respects the partially centralized approach guarantees a more reliable and efficient operating condition. Moreover, the requirement on the buffer capacitance are less tight because the d.c. link continuously receives power from some generators of the farm and, therefore, its voltage is more easily kept over the critical values.

As far as the use of each inverter is concerned, in the partially centralized approach the inverter is used constantly even if the energy carried by the seawaves is small, for the decentralized approach the inverter are used discontinuously and for a percentage of time lower than $50 \%$.

The continuity and the smoothness of the generation is enormously higher in the partially centralized approach. This is due to the fact that a common dc link can have a higher buffer capacitance and therefore it is much more stable than a dc link connected to a single generator.

\section{CONCLUSIONS}

In this paper we present an approach to the conversion of the power generated by a seawave power generator connected into a power farm composed by wind turbines and other seawave generators. The generator used is a Permanent Magnet (PM) linear generator that generates highly distorted emfs.
We propose and compare two possible ways to connect the generator to the network. One is a decentralized approach where for each generator there is a conversion subsystem that permits the direct connection of the generator to the a.c. grid, the other one is based on partially centralized approach where an a.c.-d.c. rectifier is connected to the generator and the rectifier outputs is pumped into a d.c. link that can receive the power from other units and that can supply a d.c.a.c. converter directly connected to the power grid.

We compare the performances of the two approaches in terms of ripple of the d.c. link voltage, the time of use of each inverter and the continuity of the generation and we show that the partially centralized approach is definitely more efficient and reliable of the centralized approach.

\section{REFERENCES}

[1] Brooke, J. Wave Energy Conversion. Elsevier Science, 2003.

[2] Evans, D.V. Power from Water Wave. Annual Rev Fluid Mech 1981, 13, 157-187.

[3] Falnes, J. Ocean Waves and Oscillating Systems. Cambridge University Press, 2002.

[4] Newman, J.N. Marine Hydrodynamics. MIT Press, 1977.

[5] Ross, D. Power from the Waves. Oxford University Press, 1995

[6] Shaw, R. Wave Energy - A Design Challenge. Ellis Harwood Ltd, John Wiley \& Sons, 1982.

[7] Polinder, H.; Gardner, F.; Vriesema, B. Linear PM generator for wave energy conversion in the AWS. Proceedings of the $14^{\text {th }}$ International Conference Electrical Machines, Helsinki, Finland, 2000, pp. 309-313.

[8] Mueller, M.A. Electrical generators for direct drive wave energy conversion. Proceedings of Inst. Elect. Eng. Gen. Transm. Distr, vol. 149, 2002, pp. 446-456.

[9] Trapanese, M. Un generatore lineare sincrono per lo sfruttamento dell'energia delle onde marine. In Quaderni del Dipartimento di Ingegneria Elettrica dell'Universita di Palermo, 1999.

[10] Polinder, H.; Mecrow, B.C.; Jack, A.G.; Dickinson, P.G.; Mueller, M.A. Conventionale and TPFM linear generators for direct-drive wave energy conversion. IEEE Transactions on Energy Conversion 2005, 20(1), 219-224.

[11] Leijon, M.; Bernhoff, H.; Agren, 0.; Isberg, J.; Sundberg, J.; Berg, M.; Karlsson, K.E.; Wolfbrandt, A. Multiphysics simulation of wave energy to electric energy conversion by permanent magnet linear generator. IEEE Transactions on Energy Conversion 2005, 20(1), 260-267.

[12] Cecconi, V.; Trapanese, M. An optimum design of the magnetic circuit of a PM linear electrical generator for the exploitation of sea waves. Proceedings of Power Electronics, Electrical Drives, Automation and Motion, SPEEDAM, Taormina, Italy, 2006, pp.116119.

[13] Bonanno, A.; Franzitta, V.; Muzio, F.P.; Trapanese, M. A multiphysics approach to the design of a seawave energy conversion system. Proceedings of ICEST, Singapore, 2008, pp. 2197-3001.

[14] Bonanno, A.; Franzitta, V.; Muzio, F.P.; Trapanese, M. Optimized design of a sea wave energy conversion system. Proceedings of IEEE-IECON 08, Florida, 2008, pp. 3010-3015. 